\section{Reported Differences Between Digital and Analog PET/CT Studies}

TO THE EDITOR: We read with interest the recent article by Koopman et al. entitled "Performance of Digital PET Compared with High-Resolution Conventional PET in Patients with Cancer" (1) published in the October issue of The Journal of Nuclear Medicine, describing the improved performance of the digital PET compared with the high-resolution analog PET in the detection of small lesions and image quality, which allows disease upstaging. These results are in line with our previous published data, showing superior performance of the digital PET/CT over the analog PET/CT in the detection rate and image quality in a group of oncologic (2) and nononcologic patients. In the discussion section, Koopman et al. indicated that our study showed 22 additional small lesions $(<10 \mathrm{~mm})$ on digital PET images in 100 oncologic patients. Indeed, the detection performance was even superior since in 17 out of the 100 oncologic patients, neither the digital nor the analog PET/CT revealed radiotracer uptake suggestive of malignancy (PET-negative). Therefore, the detection performance of the digital PET/CT was superior to the analog $\mathrm{PET} / \mathrm{CT}$ in detecting subcentimeter lesions $(<10 \mathrm{~mm})$ in 22 out of the 83 PET-positive patients $(26.5 \%)(P=0.05,95 \%$ confidence interval, 17.9-36.7). In addition, the authors considered invalid another previous study coming from our laboratory comparing the $\mathrm{SUV}_{\text {max }}$ between the digital PET/CT and the analog PET/CT (3) because of the differences in reconstruction parameters between both systems. It is well known that various technical and physics issues, such as detector performance, voxel size, and reconstruction parameters, influence SUV measurements. However, the aim of our study was not to evaluate the influence of such factors on SUV measurements, but to compare under standard clinical conditions the values rendered by both systems. The results of Koopman et al. (1) are again in line with our previous results showing increase of the $\mathrm{SUV}_{\max }$ in the digital PET/CT as compared with the analog PET/CT. Koopman et al. compared digital and analog PET/CT using high-resolution reconstructions for both systems, whereas our study compared digital and analog PET/CT under standard clinical conditions as provided by the vendor (digital PET using high-resolution reconstructions and analog PET using standard-resolution reconstructions).

We believe that in the next years, digital PET/CT will coexist with analog PET/CT, and differences in performance and SUV measurements must be considered in follow-up studies. Differences in clinical and research performance will guide the selection of the appropriate system for future given indications, with impact on the diagnosis and therapy assessment of oncologic and nononcologic diseases.

\section{REFERENCES}

1. Koopman D, van Dalen JA, Stevens H, et al. Performance of digital PET compared to high-resolution conventional PET in patients with cancer. $\mathrm{J} \mathrm{Nucl}$ Med. 2020;61:1448-1454

COPYRIGHT @ 2020 by the Society of Nuclear Medicine and Molecular Imaging.
2. López -Mora DA, Flotats A, Fuentes-Ocampo F, et al. Comparison of image quality and lesion detection between digital and analog PET/CT. Eur J Nucl Med Mol Imaging. 2019;46:1383-1390.

3. Fuentes-Ocampo F, López-Mora DA, Flotats A, et al. Digital vs. analog PET/CT: intra-subject comparison of the SUVmax in target lesions and reference regions. Eur J Nucl Med Mol Imaging. 2019;46:1745-1750.

Diego Alfonso López-Mora*
Albert Flotats
Francisco Fuentes-Ocampo
Ignasi Carrió
*Hospital de la Santa Creu i Sant Pau
Pare Claret 167
08025, Barcelona, Spain.
E-mail: dlopezmo@santpau.cat

Published online May 1, 2020.

DOI: 10.2967/jnumed.120.244608

\section{Flare Phenomenon in $O-\left(2-\left[{ }^{18} \mathrm{~F}\right]\right.$-Fluoroethyl)-L- Tyrosine PET After Resection of Gliomas: Potential Contribution from Postoperative Ischemia}

TO THE EDITOR: We read with great interest the recent article by Filss et al. published in The Journal of Nuclear Medicine (1). They observed a considerable increase of ${ }^{18} \mathrm{~F}$-FET uptake in $26 \%$ of subjects compared with preoperative values adjacent to the resection cavity with subsequent decrease in follow-up PET in most of the patients. The authors did not directly study the histopathology, but hypothesized that surgical intervention stimulated the metabolic activity of infiltrating tumor tissue with low metabolic activity before surgery. This is a very important study given the growing interest in using amino acid PET for target volume definition for radiotherapy beyond MRI signal abnormality $(2,3)$, and clinicians should be aware of this phenomenon especially during the first $2 \mathrm{wk}$ after surgery.

Postoperative ischemic changes after glioma resection are very common and can be diagnosed on early postoperative MRI by diffusion-weighted imaging. In a study of 104 patients with newly diagnosed and recurrent glioma (4), these postoperative ischemic lesions were identified in $31 \%$ of patients with newly diagnosed gliomas and $80 \%$ patients with recurrent gliomas (4). It is important to mention that most of these ischemic lesions were clinically silent, and only $24 \%$ of patients with newly diagnosed glioma and $48 \%$ of patients with recurrent glioma had new neurologic deficits (4). In another study of 251 glioblastoma patients who underwent surgery, postoperative infarct volume significantly correlated as an independent variable with overall survival (5), and the authors suggested that hypoxia might mediate invasive tumor growth and contribute to decreased survival (5).

A multitracer PET study to investigate amino acid accumulation in brain tissue surrounding focal ischemia demonstrated ${ }^{11} \mathrm{C}$-MET uptake not only in the core of ischemia but also in surviving brain tissue surrounding infarction likely representing alterations of amino acid transport or protein synthesis (6). Increased ${ }^{18} \mathrm{~F}$-FET uptake in subacute ischemia is also anecdotally reported by others 
$(7,8)$. In a preclinical study of focal cortical ischemia induction in rats, increased uptake of ${ }^{18} \mathrm{~F}$-FET was observed in the ischemic lesions for up to 7 days and the lesion-to-brain (L/B) ratio cutoff of 2 was exceeded in $48 \%$ of animals (9).

We believe that the high incidence of postoperative ischemic and other nonspecific changes that result in increased ${ }^{18} \mathrm{~F}$-FET uptake even in the absence of viable tumor may diminish the specificity of ${ }^{18} \mathrm{~F}$-FET PET early in the postoperative period. Filss et al. could test this hypothesis by correlating the location of postoperative ischemia on diffusionweighted images with increased ${ }^{18} \mathrm{~F}$-FET uptake. If concordance is seen, early postoperative MRI might provide important information with regard to the spatial location of ischemia compared with increased ${ }^{18}$ F-FET uptake and may warrant a delay in postoperative PET until nonspecific postoperative causes of uptake have resolved.

\section{REFERENCES}

1. Filss CP, Schmitz AK, Stoffels G, et al. Flare phenomenon in $O-\left(2-\left[{ }^{18} \mathrm{~F}\right]-\right.$ fluoroethyl)L-tyrosine PET after resection of gliomas. J Nucl Med. 2020;61:1294-1299.

2. Lohmann P, Stavrinou P, Lipke K, et al. FET PET reveals considerable spatial differences in tumour burden compared to conventional MRI in newly diagnosed glioblastoma. Eur J Nucl Med Mol Imaging. 2019;46:591-602.

3. Piroth MD, Galldiks N, Pinkawa M, et al. Relapse patterns after radiochemotherapy of glioblastoma with FET PET-guided boost irradiation and simulation to optimize radiation target volume. Radiat Oncol. 2016;11:87.

4. Gempt J, Förschler A, Buchmann N, et al. Postoperative ischemic changes following resection of newly diagnosed and recurrent gliomas and their clinical relevance. J Neurosurg. 2013;118:801-808.

5. Bette S, Wiestler B, Kaesmacher J, et al. Infarct volume after glioblastoma surgery as an independent prognostic factor. Oncotarget. 2016;7:61945-61954.

6. Jacobs A. Amino acid uptake in ischemically compromised brain tissue. Stroke. 1995;26:1859-1866.

7. Hutterer M, Nowosielski M, Putzer D, et al. $\left[{ }^{18} \mathrm{~F}\right]$-fluoro-ethyl-L-tyrosine PET: a valuable diagnostic tool in neuro-oncology, but not all that glitters is glioma. Neuro-oncol. 2013;15:341-351.

8. Pichler R, Dunzinger A, Wurm G, et al. Is there a place for FET PET in the initial evaluation of brain lesions with unknown significance? Eur J Nucl Med Mol Imaging. 2010;37:1521-1528.

9. Salber D, Stoffels G, Pauleit D, et al. Differential uptake of $\left[{ }^{18} \mathrm{~F}\right]$ FET and $\left[{ }^{3} \mathrm{H}\right] 1-$ methionine in focal cortical ischemia. Nucl Med Biol. 2006;33:1029-1035.

\section{S. Ali Nabavizadeh* Ilya M. Nasrallah Daniel A. Pryma} *Perelman School of Medicine of the University of Pennsylvania 3400 Spruce St. Philadelphia, PA 19104

E-mail: seyedali.nabavizadeh@pennmedicine.upenn.edu

Published online Jul. 9, 2020.

DOI: $10.2967 /$ jnumed.120.251116

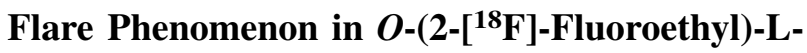 Tyrosine PET After Resection of Gliomas}

REPLY: We thank Dr. Nabavizadeh and colleagues for bringing up postoperative ischemia as a possible cause for the flare phenomenon in $O$ - $\left(2-\left[{ }^{18} \mathrm{~F}\right]\right.$-fluoroethyl)-L-tyrosine PET after resection of gliomas (1). Indeed, it cannot be excluded that postoperative ischemia has contributed to the observed flare phenomenon. On the other hand, we would like to emphasize that increased amino acid uptake in subacute ischemia is generally mild and in the range of a tumor-to-brain ratio of 2 or less. In contrast, some patients with flare phenomenon in our study (2) had maximum tumor-to-brain ratios of $O-\left(2-\left[{ }^{18} \mathrm{~F}\right]-\right.$ fluoroethyl)-L-tyrosine $\left({ }^{18} \mathrm{~F}\right.$-FET) uptake of up to 5 or above, which has not been reported in acute and subacute ischemia. According to the authors' suggestion to exclude ischemia in areas with flare phenomenon, we identified 1 patient in our database with a flare phenomenon in postoperative ${ }^{18} \mathrm{~F}$-FET PET who simultaneously underwent MR diffusion-weighted imaging (DWI) (patient 36, Fig. 1). However, the typical signs of ischemia, that is, a high signal intensity on DWI associated with low signal intensity on apparent diffusion coefficient maps, could not be identified in the corresponding area showing the flare phenomenon in postoperative ${ }^{18} \mathrm{~F}$-FET PET. Thus, DWI in this patient with a flare phenomenon does not support the "ischemia hypothesis." Nevertheless, this important aspect should be considered and further investigated in future studies.

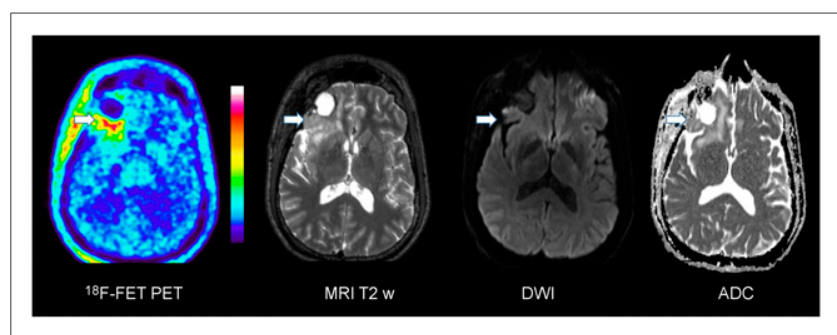

FIGURE 1. Brain scans of patient $20 \mathrm{~d}$ after resection of recurrent, right frontal oligodendroglioma World Health Organization II. ${ }^{18} \mathrm{~F}-\mathrm{FET}$ PET shows increased uptake at posterior border of resection cavity (white arrow) but no signs of ischemia, that is, a high signal intensity on diffusion-weighted imaging associated with low signal intensity on the apparent diffusion coefficient map.

\section{REFERENCES}

1. Nabavizadeh SA, Nasrallah IM, Pryma DA. Flare phenomenon in $O-\left(2-\left[{ }^{18} \mathrm{~F}\right]-\right.$ fluoroethyl)-L-tyrosine PET after resection of gliomas: potential contribution from postoperative ischemia. J Nucl Med. 2020;61:1852.

2. Filss CP, Schmitz AK, Stoffels G, et al. Flare phenomenon in $O-\left(2-\left[{ }^{18} \mathrm{~F}\right]-\right.$ fluoroethyl)L-tyrosine PET after resection of gliomas. J Nucl Med. 2020;61:1294-1299.

Published online Aug. 28, 2020.

DOI: 10.2967/jnumed.120.251819 